\title{
Data Ridden Pilots Based Estimation of Sparse Multipath Massive MIMO Channels Using Orthogonal Matching Pursuit
}

\author{
Babar Mansoor ${ }^{1}$, Moazzam Islam Tiwana ${ }^{1}$, Syed Junaid Nawaz ${ }^{1}$, Abrar Ahmed ${ }^{1}$, Abdul Haseeb ${ }^{2}$, \\ Ataul Aziz Ikram ${ }^{3}$ \\ ${ }^{1}$ Department of Electrical and Computer Engineering, COMSATS University Islamabad (CUI), \\ Islamabad, 45550, Pakistan \\ ${ }^{2}$ Department of Electrical Engineering, Institute of Space Technology (IST), \\ Islamabad, 44000, Pakistan \\ ${ }^{3}$ Department of Electrical Engineering, National University of Computer and Emerging Sciences, \\ Islamabad, 44000, Pakistan \\ babar_mansoor@comsats.edu.pk
}

\begin{abstract}
Massive Multiple-Input Multiple-Output (MIMO) is envisioned to be a strong candidate technology for the upcoming 5th generation (5G) of wireless communication networks. This research work presents a novel Compressed Sensing (CS) and Superimposed Training (SiT) based technique for estimating the sparse uplink channels in massive MIMO systems. The proposed technique involves arithmetic addition of a periodic, but low powered training sequence with each user's information sequence. Consequently, separately dedicated resources for the pilot symbols are not needed. Moreover, to attain the estimates of the Channel State Information (CSI) in the uplink, the sparsity exhibited by the MIMO channels is exploited by incorporating CS based Orthogonal Matching Pursuit (OMP) algorithm. For decoding the transmitted information symbols of each user, a Linear Minimum Mean Square Error (LMMSE) based equalizer is incorporated at the receiving Base Station (BS). Based on the obtained simulation results, the proposed SiT-OMP technique outperforms the existing Least Squares (SiT) channel estimation technique. The comparison is done using performance metrics of the Bit Error Rate (BER) and the Normalized Channel Mean Square Error (NCMSE).
\end{abstract}

Index Terms-Channel estimation; Compressed sensing; Matching pursuit algorithms; Massive multiple-input multipleoutput.

\section{INTRODUCTION}

The exponential growth in the smart phones usage and data hungry applications has led to an unprecedented increase in the data traffic. It is predicted that by the year 2020 there would be 500 -folds increase in the data usage by mobile wireless networks [1]. The major hurdle to meet this high data rate challenge is the growing scarcity of the wireless spectrum for cellular networks. The research communities around the globe are exploring new architectures and technologies in order to find viable solutions to these issues. One of the possible solutions is to use the millimetre Wave (mmWave) spectrum, which is mostly un-utilized to the date [2]. Another important solution is to exploit the spatial domain by aggressively

Manuscript received 29 November, 2018; accepted 6 March, 2019.

This paper has been supported by HEC funded research project 21-1934 (SRGP-R\&D-HEC-18). applying Multiple-Input Multiple-Output (MIMO) techniques. Massive MIMO is one of such technologies, in which a Base Station (BS) equipped with a very huge number of service antennas is used to serve a comparatively smaller number of mobile users. It is envisioned to be a prospective candidate for emerging $5 \mathrm{G}$ communication networks due to substantial improvements in energy and spectral efficiency [3], [4]. However, to take full advantage of such gains, require precise knowledge of the Channel State Information (CSI). Usually, CSI is not available a priori and is estimated by employing some appropriate channel estimation methods. Because of the channel reciprocity in Time Division Duplexed (TDD) massiveMIMO systems, channel estimation only in the uplink needs to be performed. Consequently, the obtained channel estimates can also be utilized for the downlink.

It is an established fact in the modern scientific literature that certain communications channel conditions result in a sparse Channel Impulse Response (CIR) either in delay, angular or spatial domains. The most important feature of the channel is that the CIR vector has only few non-zero delay taps as compared to its total length. Examples of such channels include, but are not limited to channels used in aeronautical communications [5], acoustic channels for underwater communications [6], and wideband channels using high frequency [7]. The wideband MIMO channels exhibit both sparsity and common support [8]. It is also established that the CIR of mmWave communication channels is also sparse [9]. The a priori knowledge about the channel sparsity may be exploited effectively for estimating CSI.

The existing research papers discuss many channel estimation techniques based on training, blind, and semiblind methods to estimate CSI of MIMO systems [10][12]. Recently, channel estimation techniques based upon Superimposed Training (SiT) have attracted a notable attention of the research community because of their noticeable advantages over the counterparts [13]-[16]. Such $\mathrm{SiT}$ based techniques utilize the spectrum more efficiently as they do not require dedicated slots for pilots. Rather a periodic and low power training sequence is directly added 
by the transmitter to the information bits. The receiver exploits periodic nature of training for CSI estimation. A multi-cell scenario is investigated in [17], where time multiplexed and SiT based pilot arrangements are compared using user sum-rate as a metric. The results demonstrate that superimposed pilot-based arrangement improves performance and has the capability for pilot decontamination in cellular MIMO systems [17].

Over the span of past decade, Compressed Sensing (CS) [18], [19] gains considerable attention as a promising technique for sensing and reconstruction of signals that exhibit sparsity in some specific domain. By exploiting the sparse nature of the signal under consideration, CS theory successfully reconstructs the signal below Nyquist rate with fewer possible measurements. Inside the CS theory, there exists a framework of CS based iterative greedy algorithms to approximate the sparse solution, e.g., Matching Pursuit (MP) [20], Orthogonal Matching Pursuit (OMP) [21], etc. In MP algorithm, the global optimal solution is approximated by formulating locally optimal choices at each iteration, such that the residual error among the selected choices is minimized. In the existing literature, CS is efficiently utilized for obtaining channel estimates in the field of wireless communications (see, e.g., [22-24]). However, to the best of authors' knowledge, a channel estimation technique utilizing spectrally efficient SiT along with CS based OMP for sparse massive-MIMO channels lacks in the existing literature. This research paper is comprised of the following novel contributions:

1. The proposal of a superimposed pilots based technique for channel estimation of uplink massive MIMO channels;

2. Considering the sparse nature of the channel vector, CS based greedy iterative OMP algorithm is exploited to obtain the channel estimates;

3. The effects of variations in the ratio between power of pilot and information sequences and the channel sparsity level are quantified by performance metric of Normalized Channel Mean Square Error (NCMSE);

4. The proposed estimation method is compared with an existing, but noteworthy SiT Least Squares (SiT-LS) [13] based method;

5. To decode the transmitted symbols, an equalizer based upon Linear Minimum Mean Square Error (LMMSE) [25], is incorporated at the receiving BS.

The rest of the proposed work is structured as follows. Section II presents the proposed system model. The details of the approach employed for generating the training sequences for each user are described in the Section IIIA. First-order statistics-based model for obtaining channel estimates is given in Section III-B. While Section III-C describes the existing SiT-LS channel estimation approach. The proposed SiT-OMP based channel estimation technique is discussed in Section III-D. In Section IV, an LMMSE equalizer for symbol detection at BS is presented. Simulation results, along with relative discussions, are given in Section V, whereas, proposed work is concluded in Section VI.

\section{SYSTEM MODEL}

The proposed system model for a multi-user MIMO system is detailed in Fig. 1. In this figure, $N$ is the transmitting mobile users, while $M$ is the number of elements in receiving antenna array. The uplink transmissions of $N$ users are assumed to propagate through a sparse MIMO channel. The channel has $L$ resolvable paths, among these $Q$ denotes the number of non-zero paths. The details of the Channel Estimator (CE) block are given in Section III. The channel estimator block uses the first order statistics [13] and OMP to estimate the CIR. After that, Training Effect Remover (TER) block is used to subtract the training sequence's effect present in the received signal. Then, the resulting signal is an input to the equalizer that estimates the information signal based upon LMMSE principal. Let $b_{n}$ represent the information sequence transmitted by $n^{\text {th }}$ user. $b_{n}$ is assumed to have zero mean and is mutually independent from the other $N-1$ users. Hence, the vector representation of $b_{n}$ is $\boldsymbol{b}_{n}=\left[b_{n}(0), b_{n}(1), \cdots, b_{n}(k-1)\right]^{*}$. The period of superimposed training sequence $\left(c_{n}\right)$ is $P$, such that $c_{n}(k)=c_{n}(k+a P)$. The vector representation of $c_{n}$ is given as $c_{n}=\left[c_{n}(0), c_{n}(1), \cdots, c_{n}(k-1)\right]^{*}$.

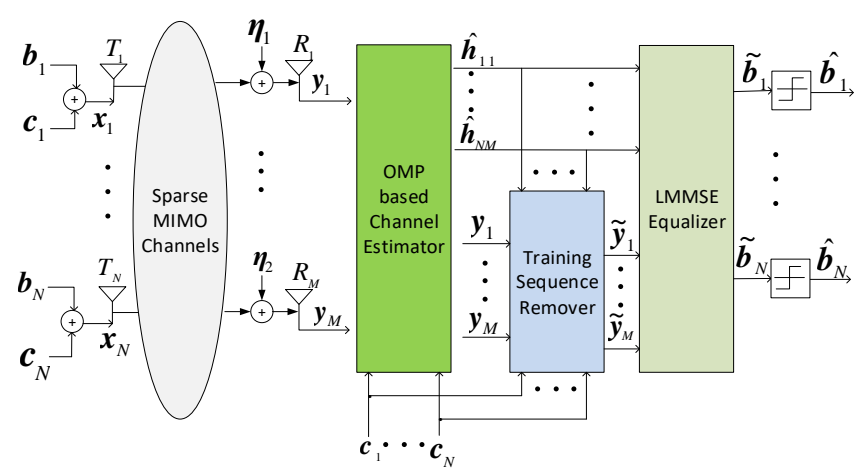

Fig. 1 Proposed system model's block diagram for uplink of massive MIMO system.

After superimposing the training over information sequence, the resulting transmit sequence $\boldsymbol{x}_{n}$, can be given by

$$
\boldsymbol{x}_{n}=\boldsymbol{b}_{n}+\boldsymbol{c}_{n}
$$

After passing through the frequency selective MIMO channel, $\boldsymbol{x}_{n}$ is received by $m^{\text {th }}$ antenna element. It is subtracted from received signal. Let $\boldsymbol{h}_{n m}=\left[h_{n m}^{0}, h_{n m}^{\ell}, \ldots, h_{n m}^{L-1}\right]^{*}$ denote the CIR between $n^{\text {th }}$ user and $m^{\text {th }}$ antenna element. If, at instant $k$, the Additive White Gaussian Noise (AWGN) appearing at an antenna element $m$ is $\eta_{m}(k)$, the signal received by this antenna element is expressed as

$$
y_{m}(k)=\sum_{n=1}^{N} \sum_{\ell=0}^{L-1} h_{n m}^{\ell} x_{n}(k-1)+\eta_{m}(k),
$$


where $y(k)$ represents the complete received signal by the $m^{\text {th }}$ antenna array at $k$ time and is given as $\boldsymbol{y}(k)=\left[y_{1}(k), y_{2}(k), \ldots, y_{M}(k)\right]^{*}$, such that

$$
\boldsymbol{y}(k)=\sum_{\ell=0}^{L-1} \boldsymbol{H}^{\ell} \boldsymbol{x}(k-\ell)+\boldsymbol{\eta}(k),
$$

where $\boldsymbol{H}^{\ell}$ is the channel matrix corresponding to the tap of $\ell^{\text {th }}$ delay tap given by

$$
\boldsymbol{H}^{\ell}=\left[\begin{array}{cccc}
h_{11}^{\ell} & h_{12}^{\ell} & \ldots & h_{1 M}^{\ell} \\
h_{21}^{\ell} & h_{22}^{\ell} & \ddots & h_{2 M}^{\ell} \\
\vdots & \vdots & \ddots & \vdots \\
h_{N 1}^{\ell} & h_{N 2}^{\ell} & \ldots & h_{N M}^{\ell}
\end{array}\right]
$$

Given

$\boldsymbol{x}(k-\ell)=\left[x_{1}(k-\ell), x_{2}(k-\ell), \cdots, x_{n}(k-\ell)\right]^{*}$ parameters

$\boldsymbol{\eta}(k)=\left[\eta_{1}(k), \eta_{2}(k), \ldots, \eta_{M}(k)\right]^{*}$, the and sampling $\quad \breve{\boldsymbol{y}}(k)=\left[y^{*}(k+L-1), \ldots, y^{*}(k)\right]^{*} \quad$ can $\quad$ be obtained as

$$
\breve{\boldsymbol{y}}(k)=\boldsymbol{H} \breve{\boldsymbol{x}}(k)+\breve{\boldsymbol{\eta}}(k),
$$

where:

$$
\begin{aligned}
\breve{\boldsymbol{x}}(k) & =\left[\boldsymbol{x}^{*}(k+L-1), \boldsymbol{x}^{*}(k+L-2), \ldots, \boldsymbol{x}^{*}(k)\right]^{*}, \\
\breve{\boldsymbol{\eta}}(k) & =\left[\boldsymbol{\eta}^{*}(k+L-1), \boldsymbol{\eta}^{*}(k+L-2), \ldots, \boldsymbol{\eta}^{*}(k)\right]^{*},
\end{aligned}
$$

and the convolution matrix, $\boldsymbol{H}$, for the sparse channels is given by

$$
\boldsymbol{H}=\left[\begin{array}{ccccc}
\boldsymbol{H}^{0} & \boldsymbol{H}^{1} & \ldots & \boldsymbol{H}^{L-1} & 0 \\
\vdots & \ddots & \ddots & \ddots & \vdots \\
0 & \boldsymbol{H}^{0} & \boldsymbol{H}^{1} & \ldots & \boldsymbol{H}^{L-1}
\end{array}\right\rfloor
$$

\section{PROPOSED OMP BASED METHOD FOR SPARSE MIMO CHANNEL ESTIMATION}

The first-order statistics used for the channel estimation, as in [13], is described in Subsection III-A and Subsection III-B. Whereas, the channel estimation based upon conventional SiT-LS and OMP are discussed in Subsection IIIC and Subsection-IIID, respectively.

\section{A. Superimposed Training Sequence Design}

Let $c_{n}(k)$ denote the training sequence assigned to the $n^{\text {th }}$ mobile user, which has a distinct cyclic frequency $P=\tilde{P} N$ where $\tilde{P} \in Z^{+}$. The $c_{n}(k)$ is represented as

$$
c_{n}(k)=\sum_{i=0}^{P-1} c_{i, n} e^{j(2 \pi i / P) k}, \quad \forall k
$$

where $j=\sqrt{-1}$. While

$$
c_{i, n}=\frac{1}{P} \sum_{k=0}^{P-1} c_{n}(k) e^{j(2 \pi i / P) k}
$$

While choosing $c_{n}(k)$, the $\tilde{P}$ coefficients are non-zero, where $\tilde{P} \in P$. The sequence $c_{n}(k)$ is computed as below

$$
c_{n}(k)=\sum_{i=0}^{\tilde{P}-1} c_{i, n}^{\prime} e^{j \alpha_{i, n} k}, \quad \forall k,
$$

where $\alpha_{i, n}=2 \pi(i N+n-1) / P$. The coefficients $c_{i, n}^{\prime}$ are selected in such way that $1 \leq n \leq N$ and $0 \leq i \leq \tilde{P}-1$. For computing $c_{n}(k)$, a base sequence $\bar{c}_{o}(k)$ is used, such that $c_{o}(k)$ has a period of $\tilde{P}$

$$
\bar{c}_{i, o}=\frac{1}{\tilde{P}} \sum_{k}^{\tilde{P}-1} \bar{c}_{0}(k) e^{j(2 \pi i / \tilde{P}) k}
$$

The sequence $\bar{c}_{1}(k)$ is defined by repeating the periodic base sequence $\bar{c}_{o}(k) N$ times. Hence, $c_{n}(k)$ for the $n^{\text {th }}$ mobile user is calculated as $c_{n}(k)=\sigma_{c_{n}} \bar{c}_{1}(k) e^{j(2 \pi / P)(n-1) k}$.

\section{B. First-Order Statistics of the Received Signal}

The $k^{\text {th }}$ received symbol at $m^{\text {th }}$ receive antenna element, denoted by $y_{m}(k)$, has the statistical expectation $E\left\{y_{m}(k)\right\}$ given by

$$
E\left\{\mathrm{y}_{m}(k)\right\}=\sum_{n=1}^{N} \sum_{i=0}^{\tilde{P}-1} \sum_{\ell=0}^{L-1} c_{i, n}^{\prime} h_{n m}^{\ell} e^{-j \alpha_{i, n} \ell} e^{j \alpha_{i, n} k}
$$

where $\quad \alpha_{i_{1}, n_{1}} \neq \alpha_{i_{2}, n_{2}} \quad$ and $\quad n_{1} \neq n_{2}, \quad$ so that $\left\{i_{1}, i_{2}\right\} \in 0,1, \ldots, P-1$. Let $\boldsymbol{d}_{n m}$ be a vector, such that $\boldsymbol{d}_{n m}=\left[d_{n m, 0}, d_{n m, 1}, \ldots, d_{n m,(\tilde{P}-1)}\right]^{*} \quad$ with $\quad$ each $\quad d_{n m, i}$ expressed as

$$
d_{n m, i}=\sum_{\ell=0}^{L-1} c_{i, n}^{\prime} h_{n m}^{\ell} e^{-j \alpha_{i, n} \ell}
$$

Let $\hat{\boldsymbol{d}}_{n m}=\left[\hat{d}_{n m, 0}, \hat{d}_{n m, 1}, \ldots, \hat{d}_{n m,(\tilde{P}-1)}\right]^{*}$ be the meansquare estimate of $\boldsymbol{d}_{n m}$ as given in [13]. The coefficients of $\boldsymbol{d}_{n m}$, can be expressed as follows

$$
\hat{d}_{n m, i}=\frac{1}{\breve{K}} \sum_{k=0}^{\breve{K}-1} y_{m}(k) e^{-j \alpha_{i, n} k},
$$

where $\breve{K}$ denotes the count of all received symbols. When 
$\breve{K} \rightarrow \infty, \quad$ accordingly $\hat{d}_{n m, i} \rightarrow d_{n m, i}$. The difference between $d_{n m, i}$ and $\hat{d}_{n m, i}$, is the error $\varepsilon_{n m, i}$. Thus, $\varepsilon_{n m, i}$ is given as

$$
\hat{d}_{n m, i}=d_{n m, i}+\varepsilon_{n m, i} .
$$

The estimation error in the calculation of $\varepsilon_{n m, i}$ results from the cumulative effect of (i) interference of superimposed training sequence of the desired user, (ii) the interference of superimposed training and information sequences of the other users, (iii) the AWGN noise. The vector form of (16) can be expressed as

$$
\hat{\boldsymbol{d}}_{n m}=\boldsymbol{C}_{n} \boldsymbol{h}_{n m},
$$

where

$$
\boldsymbol{C}_{n}=\operatorname{diag}\left\{c_{0, n}^{\prime}, c_{1, n}^{\prime}, \ldots, c_{(\tilde{P}-1), n}^{\prime}\right\} \times \boldsymbol{V}_{n} .
$$

In (18), $\boldsymbol{V}_{n}$ denotes the Vandermonde matrix.

\section{Least Squares Estimate}

From (18) the channel's least square estimate can be obtained as in [13]

$$
\hat{h}_{n m}^{\mathrm{SiT}-\mathrm{LS}}=\left(\boldsymbol{C}_{n}^{*} \boldsymbol{C}_{n}\right)^{-1} \boldsymbol{C}_{n}^{*} \hat{d}_{n m}
$$

In case of noise with non-zero mean, the channel can be calculated using the condition $\tilde{P} \geq L+1$ and removing $\hat{d}_{n m, 0}$ from $\hat{d}_{n m}$, and removing first row of $\boldsymbol{C}_{n}$.

\section{Proposed OMP Channel Estimator}

According to the existing literature, it is well established that the massive MIMO channels exhibit sparse nature in angular, delay or spatial domain. Therefore, in the uplink communication scenario of massive MIMO, the frequency selective channel between $n^{\text {th }}$ user and $m^{\text {th }}$ BS antenna at the receiver is assumed to be $Q$ sparse, i.e., $Q=\left\|h_{n m}\right\|_{\ell_{0}} \ll L$. The optimum solution to estimate the sparse channel vector from (18) is to use $\ell_{0}-$ norm, but $\ell_{0}$-norm is a computationally NP hard problem. Therefore, the proposed method exploits CS based OMP greedy iterative algorithm to obtain the estimate of sparse channel vector from (18). The optimization problem to be solved can be casted from (18) as follows

$$
\min _{\boldsymbol{h}_{n m}}\left\|\boldsymbol{C}_{n} \boldsymbol{h}_{n m}-\hat{\boldsymbol{d}}_{n m}\right\|_{\ell_{2}} \text { s. t. }\left\|\mathbf{h}_{n m}\right\|_{\ell_{0}} \leq Q
$$

The proposed SiT based channel estimation algorithm exploiting OMP is presented as follows.

Input: Matrix $\mathbf{C}_{n}$, vector $\hat{\boldsymbol{d}}_{n m}$, and sparsity $\mathrm{Q}$.

Step 1: Initialize residual vector $\boldsymbol{r}=\hat{\boldsymbol{d}}_{n m}$ and set $\Omega=\varnothing$. The set $\Omega$ contains the indices of all the columns of $\boldsymbol{C}_{n}$ that have been utilized. So, initially this set should be empty.

Step 2: While $\left\|\boldsymbol{h}_{n m}\right\|_{\ell_{0}}<Q$ (i.e., until sparsity constraint is not met).

Step 3: Compute $h_{n m, j}=\boldsymbol{c}_{n, j}^{*} \boldsymbol{r} \quad \forall j \notin \Omega$ (i.e., compute all the projections of the residual vector on to the columns of $\boldsymbol{C}_{n}$ that have not been utilized yet).

Step 4: Choose $i=\underset{j \notin \Omega}{\arg \max }\left|h_{n m, j}\right|$ (i.e., pick the largest projection).

Step 5: Update the index set $\Omega \cup\{i\}$.

Step 6: Compute $\boldsymbol{h}_{n m}^{\star}=\arg \min \left\|\boldsymbol{C}_{n, \Omega} \boldsymbol{h}_{n m}-\hat{\boldsymbol{d}}_{n m}\right\|_{\ell_{2}}^{2}$.

Step 7: Update the residual vector $\mathbf{r}=\mathbf{d}_{n m}-\mathbf{C}_{n, \Omega} \mathbf{h}_{n m}^{\star}$.

Step 8: Assign $\hat{\boldsymbol{h}}_{n m}^{\mathrm{SiT}-O M P}=\boldsymbol{h}_{n m}^{\star}$.

\section{LMMSE EQUALIZER FOR SYMBOL DETECTION}

Once the channel estimates are obtained using the proposed technique, the transmitted symbols can be detected at the BS by using an equalizer. In the proposed system model, we have incorporated an LMMSE equalizer as in [25], for this purpose. As a priori, each user's training sequence is known at the BS. The training sequence of each user is removed from the received signal as follows

$$
\tilde{y}_{m}(k)=y_{m}(k)-\sum_{n=1}^{N} \sum_{\ell=0}^{L-1} \hat{h}_{n m}^{\ell} c_{n}(k-\ell),
$$

where $\hat{h}_{n m}^{\ell}$ is $\ell^{\text {th }}$ tap of the channel vector $\boldsymbol{h}_{n m}$. After the superimposed pilots are removed from the received signal, the resultant signal is then input to the equalizer for symbol detection. The weights, $\boldsymbol{w}_{n}$, of the LMMSE equalizer, as calculated in [25], are given below

$$
\boldsymbol{w}_{n}=\left.\left(\breve{\boldsymbol{H}} \breve{\boldsymbol{H}}^{*}+2 \hat{\sigma}_{n}^{2} \boldsymbol{I}\right)^{-1} \breve{\boldsymbol{H}}\right|_{(m-1)\left(L_{e}+L-1\right)+(\tau+1)},
$$

where $L_{e}$ represents the number of equalizer taps, $\boldsymbol{I}$ is the identity matrix with dimensions $L_{e} \times L_{e}, \tau$ denotes the mapping delay, and the $i^{\text {th }}$ column of $\breve{\boldsymbol{H}}$ is represented by $\left.\breve{\boldsymbol{H}}\right|_{i}$. While $\breve{\boldsymbol{H}}$ denotes the convolutional matrix of dimensions $L_{e} \times\left(L_{e}+L-1\right)$. The estimated impulse response vectors of channels can be used to calculate $\breve{\boldsymbol{H}}_{n m}$ as follows

$$
\breve{\boldsymbol{H}}_{n m}=\left[\begin{array}{cccccccc}
\breve{h}_{n m}^{0} & \breve{h}_{n m}^{1} & \ldots & \breve{h}_{n m}^{L} & 0 & 0 & \ldots & 0 \\
0 & \breve{h}_{n m}^{0} & \breve{h}_{n m}^{1} & \ldots & \breve{h}_{n m}^{L} & 0 & \ddots & 0 \\
\vdots & \ddots & \ddots & \ddots & \ddots & \ddots & \ddots & \vdots \\
0 & 0 & \ldots & 0 & \breve{h}_{n m}^{0} & \breve{h}_{n m}^{1} & \ldots & \breve{h}_{n m}^{L}
\end{array}\right]
$$

The information sequence transmitted by $n^{\text {th }}$ user can be decoded as 


$$
\tilde{b}_{n}(k)=\sum_{m=1}^{M} \sum_{i=0}^{L_{e}-1} w_{n m, i} \tilde{y}_{m}(k-i) .
$$

A hard decision mapper is utilized to calculate the estimates $\hat{b}_{n}(k)$ from $\tilde{b}_{n}(k)$, where $\tilde{b}_{n}(k)$ are symbols modulated using Binary Phase Shift Keying (BPSK).

\section{Simulation Results}

The simulated scenario consists of a massive MIMO communication system with $N=4$ single antenna users that are communicating in uplink with a BS having $M=64$ antennas. Between the $n^{\text {th }}$ user and $m^{\text {th }}$ antenna at the BS, the channel vector $\boldsymbol{h}_{n m}$ generated for each user independent of the others. The length of the channel vector, i.e., number of resolvable multi-paths, represented by $L$, is set 14 . The channel vector $\boldsymbol{h}_{n m}$ is assumed to be $Q$ sparse, such that the channel sparsity level is $Q / L$. Each user's information sequence is assumed to be BPSK modulated with variance $\sigma_{b}^{2}$ and zero mean. The low power training sequence of $n^{\text {th }}$ user $c_{n}(k)$ is assumed to be periodic with period $P=15$. The Training-to-Information Power Ratio (TIR) equal to be $\sigma_{c}^{2} / \sigma_{b}^{2}$. The received signal is corrupted by AWGN. In the first simulation set up, the performance of proposed scheme with the change in channel's sparsity level is investigated. In this regard, the graphs for NCMSE and BER, shown in Fig. 2, are obtained for varying sparsity levels.

It is illustrated in Fig. 2 that for a Signal to Noise Ratio (SNR) of $10 \mathrm{~dB}$, an improvement of $10 \mathrm{~dB}$ in NCMSE, and for a BER of $10^{-2}$, an improvement of $6 \mathrm{~dB}$ in SNR is obtained for the proposed SiT-OMP technique for the channel estimation. The Fig. 3 investigates the effect of variations in TIR.

Figure 3 shows that the increase in the TIR power ratio results in improved channel estimates. However, there is a tradeoff between the received signal SNR and TIR as higher values of TIR leads to degradation in SNR. Hence, an optimal choice needs to be made between TIR and SNR.

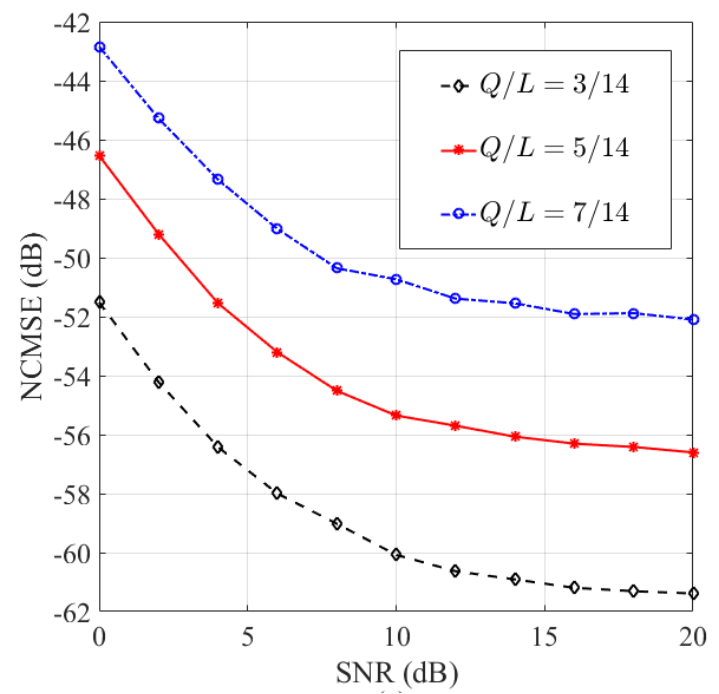

(a)

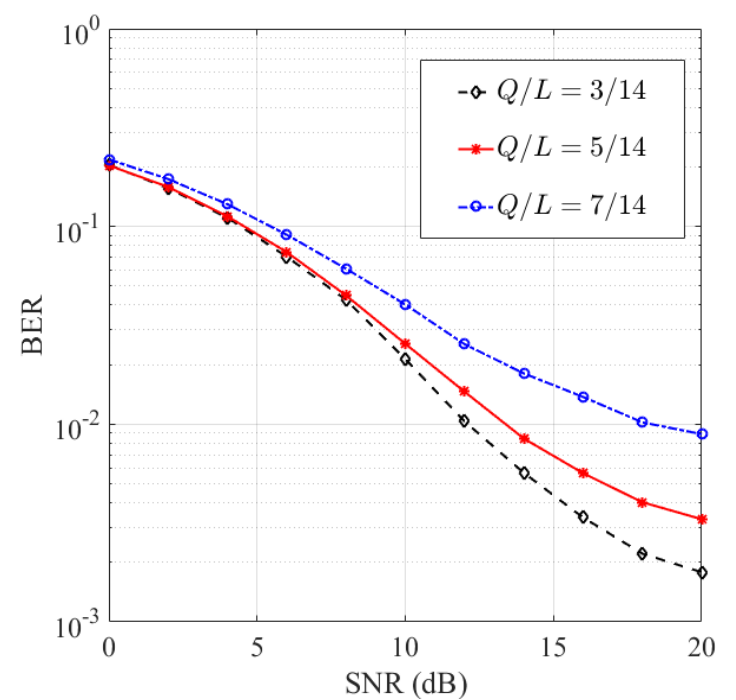

Fig. 2. Effect of variations in channel's sparsity level for SiT-OMP on NCMSE (a), on BER for $N=4, M=64, \sigma_{c}^{2} / \sigma_{b}^{2}=0.3, K=10^{3}$ and $Q / L=3 / 14,5 / 14,7 / 15$ (b).

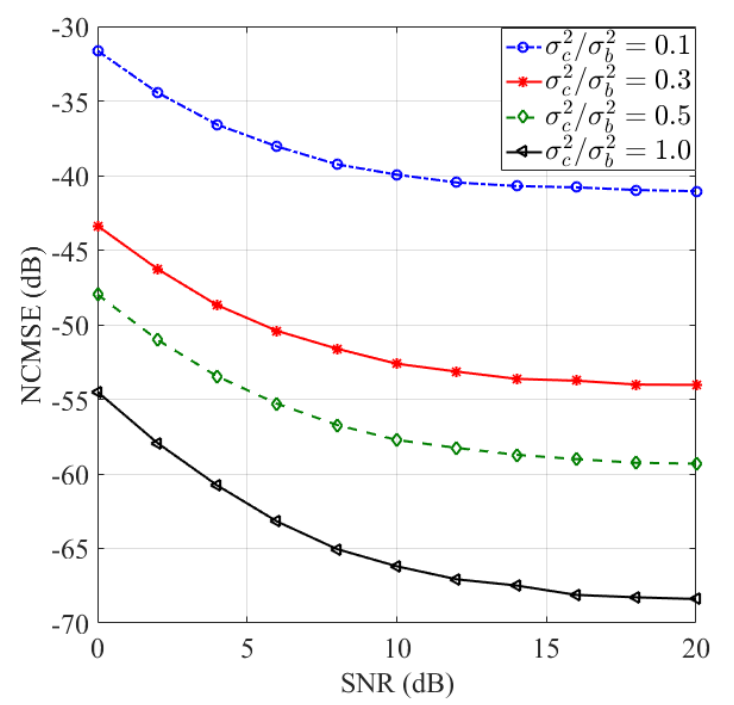

Fig. 3 Effect of variations in $\sigma_{c}^{2} / \sigma_{b}^{2}$ on the proposed SiT-OMP technique for $\quad N=4, \quad M=64, \quad Q / L=3 / 14, \quad K=10^{3}, \quad$ and $\sigma_{c}^{2} / \sigma_{b}^{2}=0.1,0.3,0.5$ and 1.0.

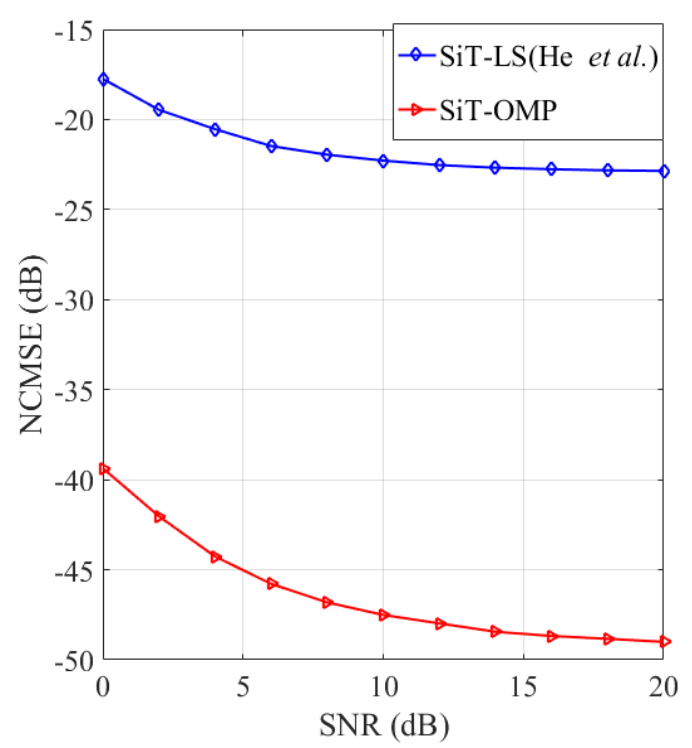

(a) 


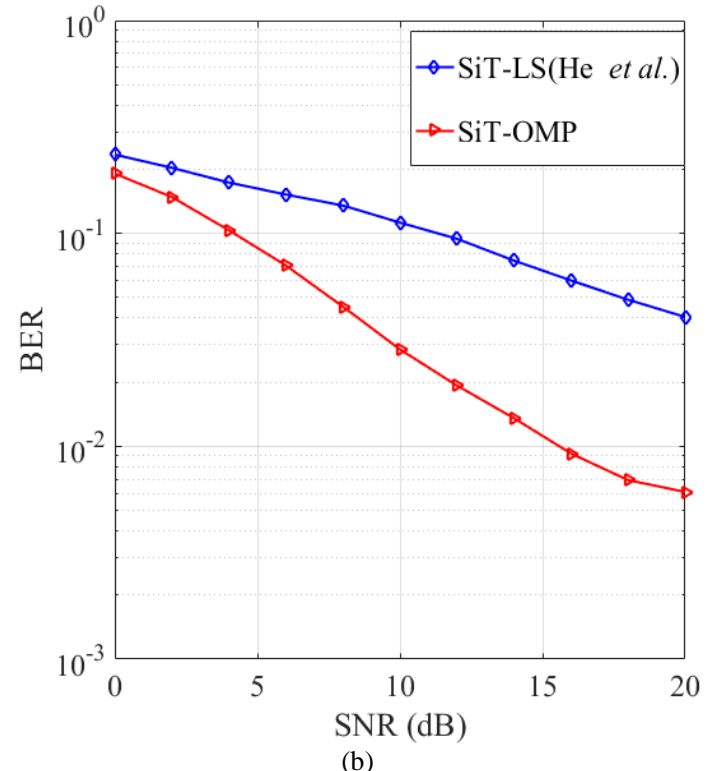

(b)

Fig 4. Performance of proposed SiT-OMP technique compared with the existing SiT-LS technique based on, (a) NCMSE (b) BER, for $N=4$,

$M=64, \quad \sigma_{c}^{2} / \sigma_{b}^{2}=0.3, \quad K=10^{3}$, and $Q / L=3 / 14$.

NCMSE and BER are set as performance metrics for performance comparison of the proposed SiT-OMP technique with the noteworthy existing SiT-LS technique for channel estimation, as shown in Fig. 4. At SNR of $10 \mathrm{~dB}$, the NCMSE of suggested SiT-OMP technique gives a performance enhancement of about $25 \mathrm{~dB}$, and at a BER of $10^{-1}$, the proposed SiT-OMP technique has a $10 \mathrm{~dB}$ performance improvement over SiT-LS. This significant enhancement in the performance is a result of considering the preceding knowledge of sparse nature of channel vector.

\section{CONCLUSIONS}

Massive MIMO systems are foreseen to play a significant part in the emerging cellular communication networks because of their impressive gains in terms of order of magnitudes increase in data rates, and energy and spectral efficiency compared to the present-day networks. However, reaping these benefits require accurate CSI estimates. In TDD based massive MIMO systems, the channel reciprocity leverages the burden of obtaining the channel estimates only in the uplink rather than both for up- and downlinks. Moreover, it is established that massive MIMO and mmWave based channels exhibit joint sparsity in delay, angular or spatial domain. This research work proposes the use of compressed sensing in conjunction to the superimposed training for channel estimation in uplink of a massive MIMO system. The proposed SiT-OMP technique exploits the sparsity in massive MIMO channels. It is evident that the suggested SiT-OMP technique, when compared with the existing least squares-based channel estimation technique, has better performance in terms of NCMSE and BER. It is demonstrated that the channel sparsity of $Q / L=3 / 14$ the proposed SiT-OMP achieves a performance gain of $25 \mathrm{~dB}$ in NCMSE and $10 \mathrm{~dB}$ in SNR for a BER of $10^{-1}$ over existing SiT-LS technique. It is also established that the proposed technique performs even better as the channel becomes sparser. Moreover, it is also discussed that there is a performance tradeoff between the TIR and SNR at the receiver. Hence, a suitable value of TIR needs to be selected for an optimum performance in SNR.

\section{REFERENCES}

[1] T. N. Nan, S. Nagata, A. Benjebbour, Y. Kishiyama, T. Hai, S. Xiaodong, Y. Ning, and L. Nan, "Trends in small cell Enhancements in LTE advanced”, IEEE Commun. Mag., vol. 51, no. 2, pp. 98-105, 2013. DOI: 10.1109/MCOM.2013.6461192.

[2] R. Haines, W. H. Chin, and Z. Fan, "Emerging technologies and research challenges for 5G wireless networks", IEEE Wireless Commun., vol. 21, no. 2, pp. 106-112, 2014. DOI: 10.1109/ MWC.2014.6812298.

[3] F. Rusek, D. Persson, B. K. Lau, E. G. Larsson, T. L. Marzetta, O. Edfor,s and F. Tufvesson, "Scaling up MIMO: Opportunities and challenges with very large arrays”, IEEE Signal Process. Mag., vol. 30, no. 1, pp. 40-60, 2013. DOI: 10.1109/MSP.2011.2178495.

[4] E. Larsson, O. Edfors, F. Tufvesson, and T. Marzetta, "Massive MIMO for next generation wireless systems", IEEE Commun. Mag., vol. 52, no. 2, pp. 186-195, 2014. DOI: 10.1109/MCOM .2014.6736761.

[5] F. K. Lee and P. J. McLane, "Design of nonuniformly spaced tapped delay-line equalizers for sparse multipath channels", IEEE Trans. On Commun., vol. 52, pp. 530-535, 2004. DOI: 10.1109/TCOMM. 2004.826351 .

[6] Z. Jun-yi, W.-x. Meng, and J. Shi-lou, "Sparse underwater acoustic OFDM channel estimation based on superimposed training", $J$. of Marine Science and Application, vol. 8, no. 1, pp. 65-70, 2009. DOI: 10.1007/s11804-009-8015-2.

[7] J. Ying, J. Zhong, M. Zhao, and Y. Cai, "Turbo equalization based on compressive sensing channel estimation in wideband HF systems", in Int. Conf. on Wireless Commun. Signal Process., 2013. DOI: 10.1109 /WCSP.2013.6677272.

[8] Y. Barbotin, A. Hormati, S. Rangan, and M. Vetterli, "Estimation of Sparse MIMO Channels with Common Support", IEEE Trans. On Commun., vol. 60, no. 12, pp. 3705-3716, 2012. DOI: 10.1109/TCOMM.2012.091112.110439.

[9] A. Alkhateeb, O. E. Ayach, G. Leus, and R. W. Heath, "Channel estimation and hybrid precoding for millimeter wave cellular systems", IEEE J. of Sel. Topics in Signal Process., vol. 8, no. 5, pp. 831-846, 2014. DOI: 10.1109/JSTSP.2014.2334278.

[10] S. Shahbazpanahi, A. B. Gershman, and J. H. Manton, "Closed-form blind MIMO channel estimation for orthogonal space-time block codes", IEEE Trans. On Signal Process., vol. 53, no. 12, pp. 45064517, 2005. DOI: 10.1109/TSP.2005.859331.

[11] Y. Zeng, A. R. Leyman, and T. S. Ng, "Joint semiblind frequency offset and channel estimation for multiuser MIMO-OFDM uplink", IEEE Trans. On Commun., vol. 55, no. 12, pp. 2270-2278, 2005. DOI: 10.1109/TCOMM.2007.910637.

[12] C. Fragouli, N. Al-Dhahir, and W. Turin, "Training-based channel estimation for multiple-antenna broadband transmissions", IEEE Trans. On Wireless Commun., vol. 2, no. 2, pp. 384-391, 2003. DOI: 10.1109/TWC.2003.809454.

[13] S. He, J. K. Tugnait, and X. Meng, "On superimposed training for MIMO channel estimation and symbol detection", IEEE Trans. On Signal Process., vol. 55, no. 6, pp. 3007-3021, 2007. DOI: 10.1109/TSP.2007.893941.

[14] S. J. Nawaz, K. I. Ahmed, M. N. Patwary, and N. M. Khan, "Superimposed training-based compressed sensing of sparse multipath channels", IET Commun., vol. 18, no. 6, pp. 3150-3156, 2012. DOI: 10.1049/iet-com.2012.0162.

[15] B. Mansoor, S. J. Nawaz, B. Amin, S. K. Sharma, and M. N. Patwary, "Superimposed training based estimation of sparse MIMO channels for emerging wireless networks", in Proc. 23rd Int. Conf. on Telecommun. (ICT), 2016. DOI: 10.1109/ICT.2016.7500477.

[16] B. Mansoor, S. J. Nawaz, and S. M. Gulfam, "Massive-MIMO sparse uplink channel estimation using implicit training and compressed sensing", Applied Sciences, vol. 7, no. 1, 2017. DOI: 10.3390/app7010063.

[17] K. Upadhya, S. A. Vorobyov, and M. Vehkapera, "Superimposed pilots are superior for mitigating pilot contamination in massive MIMO,” IEEE Trans. On Signal Process., vol. 65, no. 11, pp. 29172932, 2017. DOI: 10.1109/TSP.2017.2675859.

[18] E. J. Candes and T. Tao, "Decoding by linear programming", IEEE Trans. On Inf. Theory, vol. 51, no. 12, pp. 4203-4215, 2005. DOI: 10.1109/TIT.2005.858979.

[19] D. L. Donoho, "Compressed sensing", IEEE Trans. On Inf. Theory, vol. 52, no. 4, pp. 1289-1306, 2006. DOI: 10.1109/TIT.2006.871582. 
[20] S. G. Mallat and Z. Zhang, "Matching pursuits with time-frequency dictionaries", IEEE Trans. On Signal Process., vol. 41, no. 12 , pp. 3397-3415, 1993. DOI 10.1109/78.258082.

[21] G. Davis, S. Mallat, and M. Avellaneda, "Adaptive greedy approximations," J. of Constructive Approximation, vol. 13, no. 1, pp. 57-98, 1997. DOI 10.1007/BF02678430.

[22] W. U. Bajwa, J. Haupt, A. M. Sayeed, and R. Nowak, "Compressed channel sensing: A new approach to estimating sparse multipath channels", in Proc. of the IEEE, vol. 98, no. 6, pp. 1058-1076, 2010 DOI: 10.1109/JPROC.2010.2042415.

[23] G. Taubock, F. Hlawatsch, D. Eiwen, and H. Rauhut, "Compressive estimation of doubly selective channels in multicarrier systems:
Leakage effects and sparsity-enhancing processing", IEEE J. of Sel. Topics in Signal Process., vol. 4, no. 2, pp. 255-271, 2010. DOI 10.1109/JSTSP.2010.2042410

[24] X. Rao and V. K. N. Lau, "Compressive sensing with prior support quality information and application to massive MIMO channel estimation with temporal correlation", IEEE Trans. Signal Process., vol. 63, no. 18, pp. 4914-4924, 2015. DOI 10.1109/TSP.2015.2446444.

[25] S. Chen, A. Livingstone, and L. Hanzo, "Minimum bit-error rate design for space-time equalization-multiuser detection", IEEE Trans. on Commun., vol. 54, no. 5, pp. 824-832, 2006. DOI 10.1109/JPROC.2010.2042415. 